\title{
Operational Experience and Performance with the ATLAS Pixel Detector at the Large Hadron Collider at CERN
}

\author{
Xiaotong Chu*, on behalf the ATLAS Collaboration \\ Institute of High Energy Physics, Chinese Academy of Sciences \\ University of Chinese Academy of Sciences \\ E-mail: xiaotong.chu@cern.ch
}

\begin{abstract}
The tracking performance of the ATLAS detector at the Large Hadron Collider (LHC) relies critically on its 4-layer Pixel Detector, located at the core of the ATLAS tracker. The ATLAS pixel detector consists of four barrel layers and a total of six disk layers, three at each end of the barrel region. It has undergone significant hardware and readout upgrades to meet the challenges imposed by the higher collision energy, pileup and luminosity that are delivered by the LHC. The key status and performance metrics of the ATLAS Pixel Detector are summarised, and the operational experience and requirements to ensure optimum data quality and data taking efficiency will be described, with special emphasis on radiation damage experience. By the end of the proton-proton collision runs in 2018, the IBL had received an integrated fluence of approximately $\phi=9 \times 10^{14} 1 \mathrm{MeV}$ neq $/ \mathrm{cm}^{2}$. The innermost of the three outer layers (B-layer) has been exposed to about half the fluence of the IBL, and lower fluences for other layers. The ATLAS collaboration is continually evaluating the impact of radiation on the Pixel Detector. In particular, signs of degradation are visible but are not impacting yet the tracking performance, including a trend of decreasing charge collection, $\mathrm{dE} / \mathrm{dX}$, occupancy reduction with integrated luminosity, underdepletion effects with IBL, effects of annealing that are significant for the inner-most layers. A quantitative analysis of all these effects will be presented and discussed, as well as the operational issues and mitigation techniques adopted during the LHC run and the ones foreseen during the LHC Long Shutdown 2.
\end{abstract}

European Physical Society Conference on High Energy Physics - EPS-HEP2019 -

10-17 July, 2019

Ghent, Belgium

${ }^{*}$ Speaker. 


\section{Introduction}

ATLAS [1] is one of the four major experiments at the Large Hadron Collider (LHC) at CERN. It is a general-purpose particle physics experiment designed to exploit the full discovery potential that the LHC provides.

The tracking performance of the ATLAS detector relies critically on its Pixel Detector [2], located at the core of the ATLAS tracker. The ATLAS Pixel detector consists of four barrel layers: Insertable B-Layer (IBL) [3], B-Layer, Layer 1, Layer 2, and two end-caps containing three Disk layers each, as illustrated in Figure 1 [4].
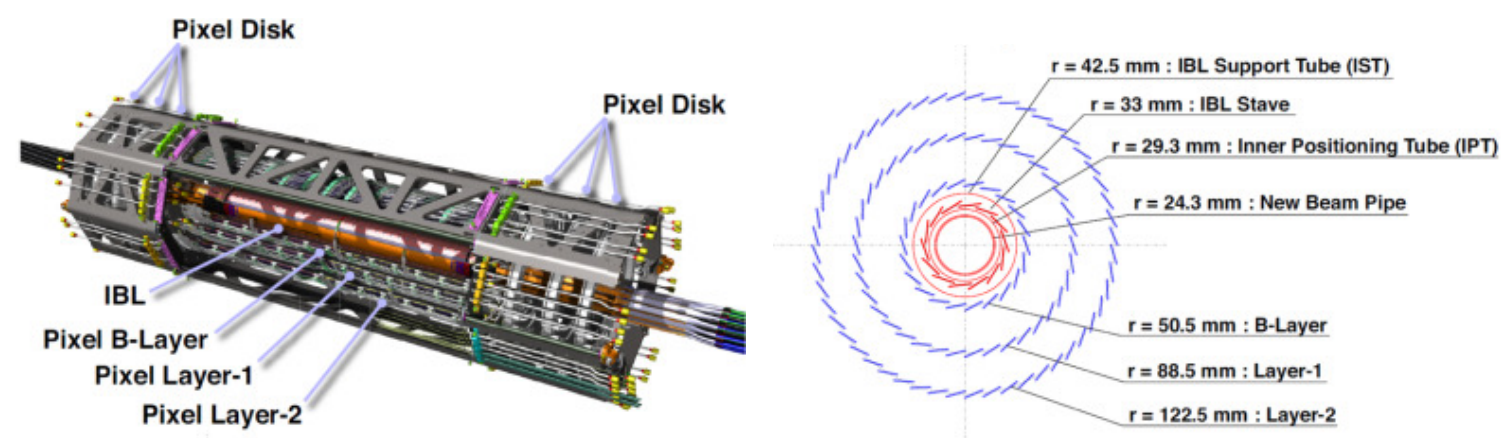

Figure 1: Layout of the ATLAS Pixel detector [4]. Left figure: 3D model of the barrel layers and end-cap disks. Right figure: transverse layout and radial position of the barrel layers.

\begin{tabular}{lcc}
\hline & FE-I3 & FE-I4 \\
\hline Pixel size $\left[\mu \mathrm{m}^{2}\right]$ & $50 \times 400$ & $50 \times 250$ \\
Pixel array & $18 \times 160$ & $80 \times 336$ \\
Chip size $\left[\mathrm{mm}^{2}\right]$ & $7.6 \times 10.8$ & $20.2 \times 19.0$ \\
No. of transistors [millions] & 3.5 & 87 \\
Analog/digital current $[\mu \mathrm{A} / \mathrm{pix}]$ & $26 / 17$ & $10 / 10$ \\
Analog/digital voltage $[\mathrm{V}]$ & $1.6 / 2.0$ & $1.5 / 1.2$ \\
\hline
\end{tabular}

Table 1: Specifications of FE-I3 and FE-I4 front-end chips [2][6].

The IBL was installed during Long Shutdown 1 (LS1) [5]. It consists of $200 \mu \mathrm{m} \mathrm{n}+-\mathrm{in}-\mathrm{n}$ planar silicon sensors and $230 \mu \mathrm{m} n+-i n-p$ 3D silicon sensors, read-out by front-end (FE) chip FE-I4 [4][6]. The outer pixel layers, installed in 2007, consist of $250 \mu \mathrm{m} n+-i n-n$ planar silicon sensors connected to FE-I3 chips [2]. Table 1 lists the specifications of the two kinds of frontend chips [2][6]. The designed radiation hardness is $50 \mathrm{Mrad}$ and $250 \mathrm{Mrad}$ for FE-I3 and FE-I4 respectively.

\section{Radiation damage effects}

Figure 2 shows the simulated $1 \mathrm{MeV}$ equivalent neutron fluence absorbed by the barrel layers of the ATLAS Pixel detector at $\eta=0$ during Run 2 [7]. Two different types of radiation damage, described in the following sections, are visible in the Pixel detector: non-ionizing damage to the sensor bulk and ionizing damage to the front-end chip [8]. 

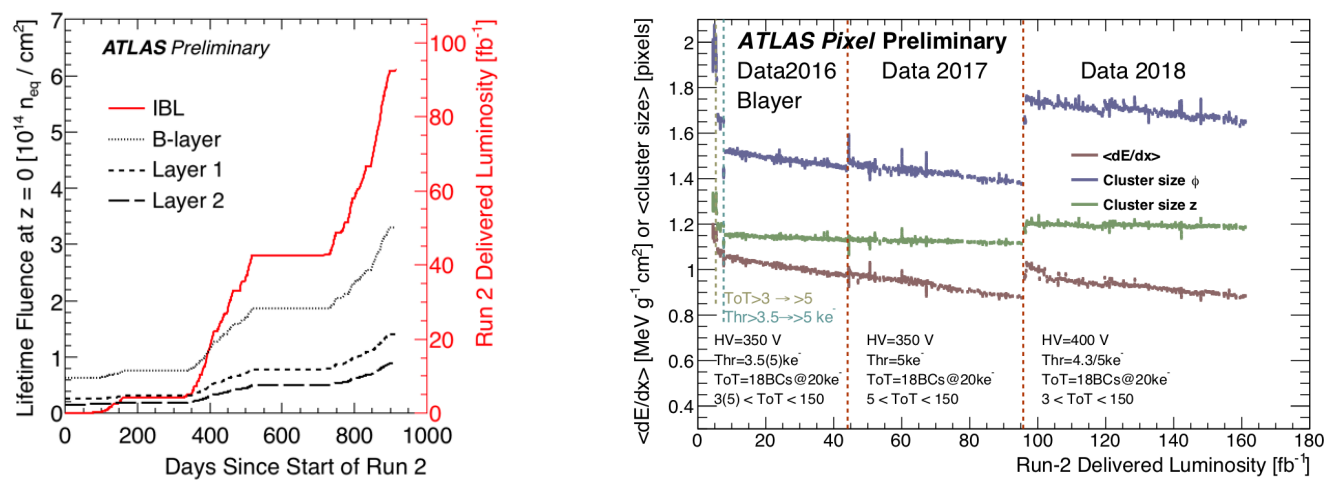

Figure 2: Si $1 \mathrm{MeV}$ equivalent neutron flu- Figure 3: Average cluster size (in $\phi$ and $\mathrm{z}$ ) and $\mathrm{dE} / \mathrm{dx}$ as a ence (neq $/ \mathrm{cm}^{2}$ ) at $\eta=0$ during Run 2 [7]. function of delivered luminosity during Run 2 for B-Layer [7].

\subsection{Radiation damage in sensor bulk}

Radiation damage in the sensor bulk affects the electric field profile and the charge collection efficiency (Figure 4) [7]; it affects the depletion voltage and generates charge trapping affecting collected charge and dE/dX. Figure 3 shows the gradual decrease of the measured $\mathrm{dE} / \mathrm{dx}$ and cluster size of the B-Layer as a function of delivered luminosity in Run 2 [7]. On the other hand, changes in the electric field profile affects the cluster charge distribution.

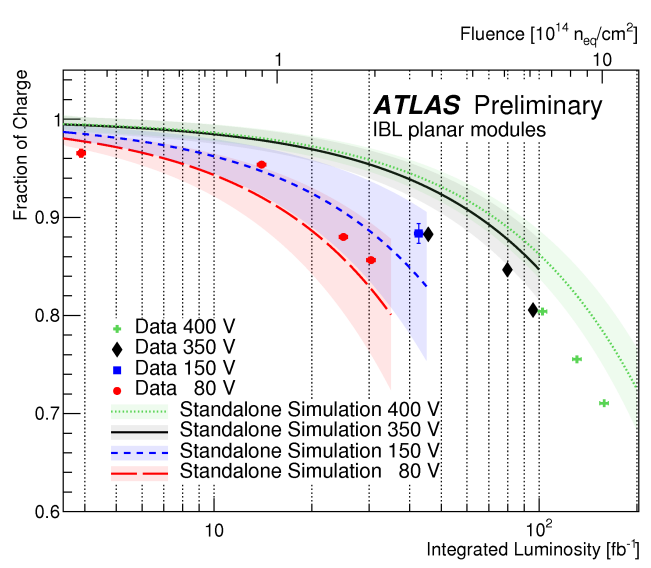

Figure 4: Charge collection efficiency for IBL planar modules as a function of integrated luminosity [7].

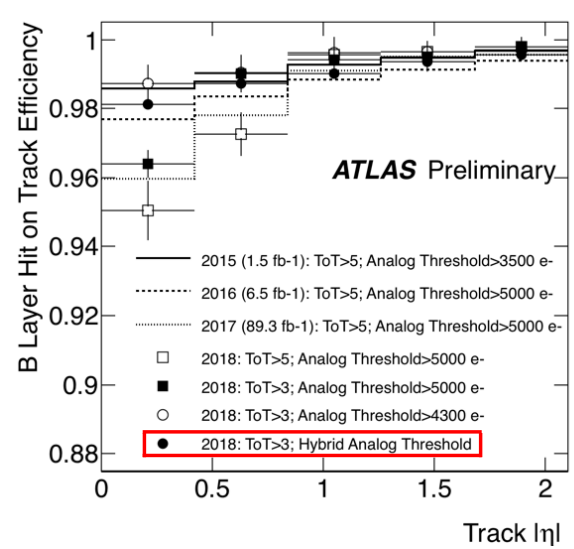

Figure 5: Efficiency for B-Layer hits associated to a reconstructed track as a function of $|\eta|[7]$.

Bias voltage values in different layers are regularly increased to partially recover the depletion area and charge collection efficiency [7]. The analog and digital thresholds were reduced in 2018 to compensate for the loss of charge collection efficiency due to radiation damage, as marked in Figure 5 [7].

\subsection{Radiation damage in the front-end chip}

Radiation damages on $\mathrm{SiO}_{2}$ and $\mathrm{SiO}_{2}$-Si induce trapped charges which increase low-voltage leakage current in FE-I4. IBL low-voltage leakage current increases due to radiation damage at low 
total ionizing dose (TID). Moreover, it causes drift on the analog threshold and time over threshold (ToT) of the FE-I4. This requires regular tunings to bring the mean back to the target value and reduce the RMS. Figure 6 shows the ToT and its RMS evolution of IBL during 2018 [9].

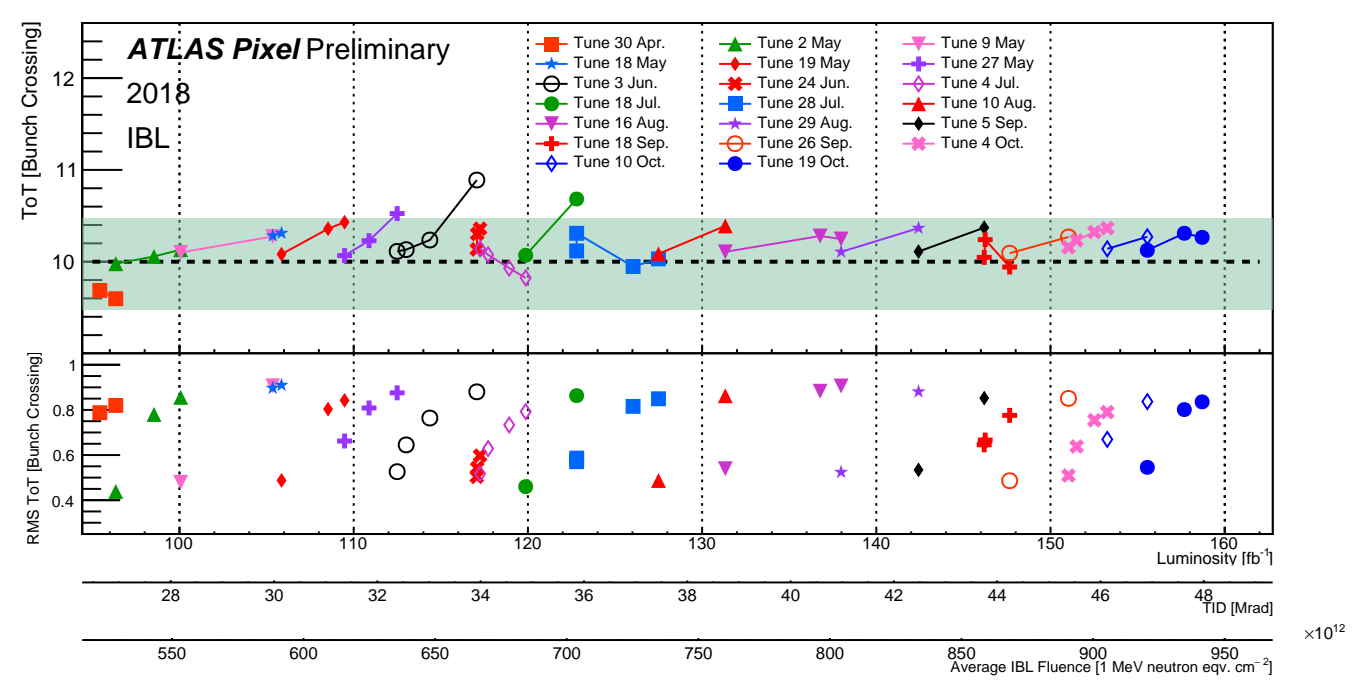

Figure 6: Evolution of mean and RMS of ToT over all pixels in IBL measured in calibration scans. Each color/symbol series corresponds to a single tuning of the detector [9].

\section{Detector performance}

The tracking performance has been significantly improved since the insertion of the IBL. Figure 7 indicates that the impact parameter resolution $\left(\sigma_{\mathrm{d}_{0}}\right)$ is improved nearly by a factor of two at low $p_{\mathrm{T}}$ as a result of adding the IBL point measurement at smaller radius and with high spatial resolution [10]. Figure 8 shows the IBL spatial resolution in Run 2 [7], measured from the corrected transverse positions of two reconstructed IBL clusters associated to a charged particle track in the module overlap region. The spatial resolution $(\sim 10 \mu \mathrm{m}$ in $\mathrm{r}-\phi)$ is found to slightly degrade over the period of Run 2 as a result of the decrease of collected charge and change in Lorentz angle ${ }^{1}$.

\section{Conclusion}

Thanks to the tremendous effort of the operation team, the ATLAS Pixel Detector has been operated successfully and has delivered excellent performance and data quality despite the increasing luminosity of the LHC during Run 2, in which the overall performance has been improved further thanks to the installation of IBL. The radiation damage effects are visible in several observables, but have no significant impact on the quality of reconstructed physics objects. The ATLAS Pixel community is preparing towards Run 3.

\footnotetext{
${ }^{1}$ The Lorentz angle is given by the deflection of the charge carriers in a magnetic field perpendicular to the electric field [11].
} 

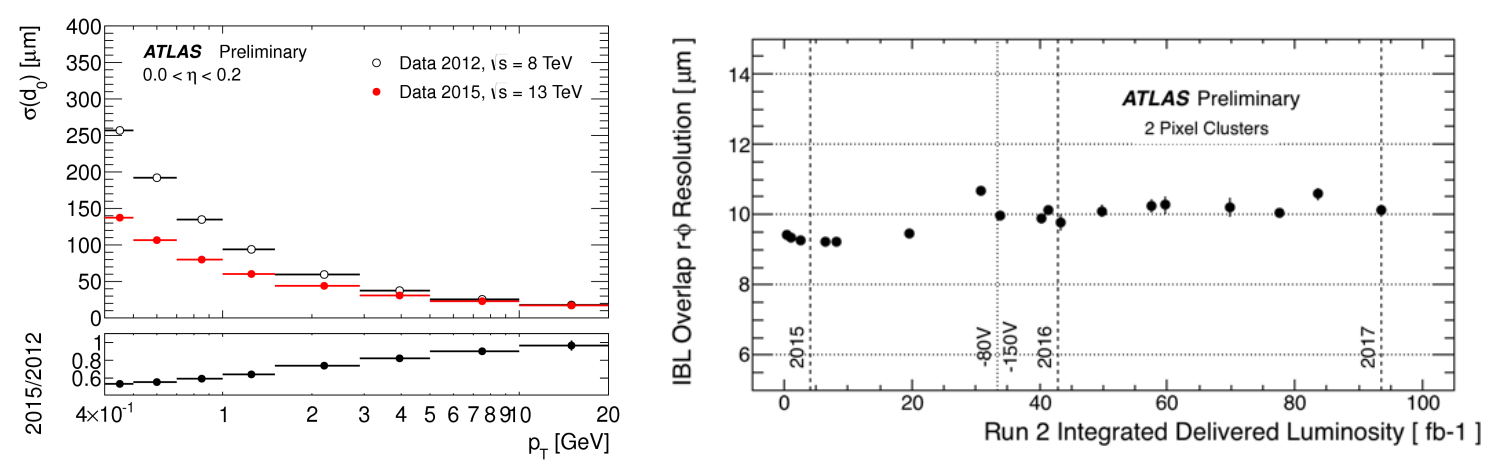

Figure 7: Unfolded transverse impact pa- Figure 8: Spatial resolution of IBL hits associated to reconrameter resolution measured from data in structed particle tracks in di-jet events as a function of the in2015 including the IBL, in contrast with tegrated luminosity [7].

2012 data (no IBL) [10].

\section{References}

[1] ATLAS Collaboration, The ATLAS Experiment at the CERN Large Hadron Collider, 2008 JINST 3 S08003.

[2] G. Aad et al., ATLAS pixel detector electronics and sensors, JINST 3 (2008) P07007. doi:10.1088/1748-0221/3/07/P07007.

[3] B. Abbott, et al., Production and Integration of the ATLAS Insertable B-Layer, JINST 13 (05) (2018) T05008 [arXiv:1803.00844] doi:10.1088/1748-0221/13/05/T05008.

[4] M. Backhaus, The upgraded Pixel Detector of the ATLAS Experiment for Run 2 at the Large Hadron Collider, NIMA Volume 831, 2016, Pages 65-70.

[5] ATLAS Collaboration, ATLAS Insertable B-Layer Technical Design Report, CERN-LHCC-2010-013, ATLAS-TDR-19 [https://cds.cern.ch/record/1291633].

[6] FE-I4 Collaboration, The FE-I4B Integrated Circuit Guide, Version 2.32012154 [https://indico.cern.ch/event/261840/contributions/1594374/attachments/462649/641213/FEI4B_V2.3.pdf].

[7] ATLAS Collaboration, Public Pixel Tracker Plots for Collision Data, https://twiki.cern.ch/twiki/bin/view/AtlasPublic/PixelPublicResults.

[8] ATLAS Collaboration, Radiation induced effects in the ATLAS Insertable B-Layer readout chip, ATL-INDET-PUB-2017-001 [https://cds . cern.ch/record/2291800].

[9] ATLAS Collaboration, Cosmics and Calibration Approved Pixel Plots, https://twiki.cern.ch/twiki/bin/view/AtlasPublic/ApprovedPlotsPixel.

[10] ATLAS Collaboration, Impact Parameter Resolution, https: / / atlas.web. cern. ch/Atlas/GROUPS / PHYS ICS /PLOTS / IDTR-2015-007/.

[11] ATLAS Collaboration, Modeling the Mobility and Lorentz angle for the ATLAS Pixel Detector, ATL-INDET-PUB-2018-001. [https : / / cds . cern. ch/record/2629889]. 